Pacific Journal of Mathematic 


\title{
p-ADIC OSCILLATORY INTEGRALS AND WAVE FRONT SETS
}

\author{
D. B. HeIFETZ
}

For $K$ a $p$-adic field, we examine oscillatory integrals

$$
I(\phi, p)(\lambda)=\int_{K^{n}} \phi(x) \Psi(\lambda p(x)) d x
$$

where $\phi$ is a Schwartz function on $K^{n}, \Psi$ is an additive character, $\lambda \in K^{x}$, and $p: K^{n} \rightarrow K$ is locally analytic. If $D p \neq 0$ on the support of $\phi, \lambda \mapsto I(\phi, p)(\lambda)$ has bounded support. If $D p\left(x_{0}\right)=0$ at exactly one point $x_{0}$ in the support of $\phi$ but $D^{2} p\left(x_{0}\right)$ is non-degenerate, then

$$
I(\phi, p)(\lambda)=|\lambda|^{-n / 2} \gamma \Psi\left(p\left(x_{0}\right)\right)\left|\operatorname{det} D^{2} p\left(x_{0}\right)\right|^{-1 / 2} \phi\left(x_{0}\right)
$$

for sufficiently large $|\lambda|$, where $\gamma$ is a complex eighth root of unity. An invariant definition of wave front set, $W F_{\Lambda}(u)$, for distributions $u$ relative to an open subgroup $\Lambda$ of $K^{\times}$is proved to exist, analogous to the classical case, with "rapidly decreasing" replaced by "bounded support". Definitions of pull backs and push forwards of distributions, distribution products, and kernel maps are made, again similar to the classical case, and their wave front sets computed. Wave front sets $W F_{\Lambda}(\rho)$ of representations $\rho$ of $p$-adic groups are also defined (cf. Howe, Automorphic forms, representation theory, and arithmetic, Tata Inst., 1979, for the Lie group analogue). For admissible representations $\rho$ of, say, a semi-simple group $G$, with character $\chi_{\rho}$, we show that $W F_{\Lambda}^{0}(\rho)=W F_{\Lambda}^{0}\left(\chi_{\rho}\right)$, where $W F_{\Lambda}^{0}(\cdot) \subseteq \operatorname{Lie}(G)$ is $W F_{\Lambda}(\cdot)$ above the identity element. Functorial properties of $W F_{\Lambda}(\rho)$ are developed and examples computed.

Introduction. The motivation for this work is to apply to $p$-adic groups the approach of Howe $[\mathbf{H}]$ who has applied classical wave front set theory to Lie group representations. Chapter I develops a stationary phase formula for $p$-adic oscillatory integrals. In Chapter II, $p$-adic wave front sets are defined, relative to multiplicative subgroups of the multiplicative group of a $p$-adic field. The wave front set of a representation of a $p$-adic group is then defined and developed in Chapter III.

We denote by $K$ a locally compact field of characteristic 0 with valuation $|\cdot|_{K}, O_{K}$ its ring of integers, and $P_{K}$ the unique maximal prime ideal of $O_{K}$. Denote by $\bar{\omega}$ a fixed uniformizing element. If $|\bar{\omega}|_{K}=q^{-1}$, define $\operatorname{Ord}(x), x \in K^{\times}$, by $|x|=q^{-\operatorname{Ord}(x)}$. On the $n$-dimensional space $K^{n}$, we use the norm $\left|\left(x_{1}, \ldots, x_{n}\right)\right|_{K^{n}}=\max \left\{\left|x_{i}\right|_{K}\right\}$. The unit sphere is denoted $\Sigma^{n-1}$. Identify $K^{n}$ with its dual $\left(K^{n}\right)^{*}$ by the symmetric bilinear form $\{x, y\}=\sum_{i=1}^{n} x_{i} y_{i}$. Fix an additive character $\Psi$ of $K$ with conductor $O_{K}$. 
Any additive character of $K^{n}$ is then of the form $x \mapsto \Psi(\langle x, y\rangle)$. Taking $d x$ to be a Haar measure on $K^{n}$, the Fourier transform is defined by

$$
\hat{\phi}(y)=\int_{K^{n}} \phi(x) \Psi(\langle x, y\rangle) d x
$$

for $\phi \in L^{1}\left(K^{n}\right)$, and can be extended to $L^{2}\left(K^{n}\right)$ where, if $\int_{O_{K}} d x=1$, we have $\hat{\hat{\phi}}(x)=\tilde{\phi}(x)=\phi(-x)$.

For $X$ an analytic $n$-dimensional $K$ manifold, let $H(X)$ denote the set of locally analytic functions on $X$. By $C^{\infty}(X)$, we denote the space of locally constant complex valued functions on $X, S(X)$ the subspace of compactly supported functions in $C^{\infty}(X)$, and $S^{\prime}(X)$ the space of all linear functionals on $S(X)$. If $\omega$ is a fixed strictly positive smooth density on $X, C^{\infty}(X)$ is then identified with a subset of $S^{\prime}(X)$ by $f \mapsto f \omega$. By $T^{*} X$ we denote the analytic co-tangent space on $X$.

1. $p$-adic oscillatory integrals. For $\phi \in S(X), X \subseteq K^{n}$ open, and $p \in H\left(X \times K^{r}\right)$ let

$$
I_{\eta}(p, \phi)(\lambda):=\int_{X} \phi(x) \Psi(\lambda p(x, \eta)) d x, \quad \lambda \in K^{\times} .
$$

We compute asymptotic expansions for $\lambda \mapsto I_{\eta}(p, \phi)(\lambda)$ in the two cases where $\operatorname{grad}_{x} p$ is non-zero on $\operatorname{supp}(\phi)$, and where $p$ has one non-degenerate critical point on $\operatorname{supp}(\phi)$.

Proposition 1.1. Let $X \subseteq K^{n}$ and $V \subseteq K^{r}$ be open. Suppose that $p \in H(X \times V), \quad \phi \in S(X)$, and $\left|\operatorname{grad}_{x} p(x, \eta)\right| \geq \delta>0$ for $(x, \eta) \in$ $\operatorname{supp}(\phi) \times V$. Suppose further that $|R(x, y, \eta)|$ is bounded for $x, x+y \in$ $\operatorname{supp}(\phi)$, and $\eta \in V$, where $R$ is defined by

$$
p(x+y, \eta)=p(x, \eta)+\left\langle\operatorname{grad}_{x} p(x, \eta), y\right\rangle+\langle R(x, y, \eta) y, y\rangle .
$$

Then $\lambda \mapsto I_{\eta}(p, \phi)(\lambda)$ has bounded support on $K^{\times}$, with bound independent of $\eta \in V$.

Proof. Assume $n=1$, the proof for $n>1$ being analogous. Since $U=\operatorname{supp}(\phi)$ is open-compact, there is an integer $N$ such that if $m>N$,

$$
U=\bigcup_{i \in I} x_{i}+P_{K}^{m}
$$

for some finite set $\left\{x_{i}\right\}_{i \in I} \subset \operatorname{supp}(\phi)$. Suppose

$$
q^{a}=\inf _{x \in U, \eta \in V}\left|\operatorname{grad}_{x} p(x, \eta)\right|, \quad q^{b}=\sup _{\substack{x \in U, \eta \in V \\ x+y \in U}}|R(x, y, \eta)|,
$$

and let $M_{U}=q^{\max \{b-2 a, 2 N-b\}}$. 
If $|\lambda|>M_{U}$, then

$-\operatorname{Ord}(\lambda)>b-2 a$ and $-\operatorname{Ord}(\lambda)+a>(-\operatorname{Ord}(\lambda)+b) / 2$.

Choose $m_{0}$ such that $-\operatorname{Ord}(\lambda)+a>m_{0} \geq(-\operatorname{Ord}(\lambda)+b) / 2$. Note that $m_{0}>N$, since $-\operatorname{Ord}(\lambda)>2 N-b$.

Supposing $\phi \equiv 1$ on $U$, we have

$$
I_{\eta}(p, \phi)(\lambda)=\sum_{i \in I} \int_{P_{K}^{m_{0}}} \Psi\left(\lambda p\left(x_{i}+x, \eta\right)\right) d x
$$

for a finite set $\left\{x_{i}\right\}_{i \in I} \subset U$. Now for each $i \in I$,

$$
\begin{aligned}
\int_{P_{K}^{m_{0}}} & \Psi\left(\lambda p\left(x_{i}+x, \eta\right)\right) d x \\
= & \int_{P_{K}^{m_{0}}} \Psi\left(\lambda\left\{p\left(x_{i}, \eta\right)+\frac{d p}{d x}\left(x_{i}, \eta\right)+R\left(x_{i}, x, \eta\right) x^{2}\right\}\right) d x \\
= & \Psi\left(\lambda p\left(x_{i}, \eta\right)\right) \int_{P_{K}^{m_{0}}} \Psi\left(\lambda \frac{d p}{d x}\left(x_{i}, \eta\right) x\right) \cdot \Psi\left(\lambda R\left(x_{i}, x, \eta\right)^{2}\right) d x
\end{aligned}
$$

For $x \in P_{K}^{m_{0}},\left|\lambda R\left(x_{i}, x, \eta\right) x^{2}\right| \leq|\lambda| q^{-2 m_{0}+b} \leq 1$, hence $\Psi\left(\lambda R x^{2}\right)=1$. If $|x|=q^{-m_{0}}$ then

$$
\left|\lambda \frac{d p}{d x}\left(x_{i}, \eta\right) x\right| \geq|\lambda| q^{a-m_{0}}>1,
$$

thus $x \mapsto \Psi\left(\lambda d p\left(x_{i}, \eta\right) x / d x\right)$ is a non-trivial character on $P_{K}^{m_{0}}$. Returning to (1.1), if $|\lambda|>M_{U}$, for any $\eta \in V$,

$\int_{P_{K}^{m_{0}}} \Psi\left(\lambda p\left(x_{i}+x, \eta\right)\right) d x=\Psi\left(\lambda p\left(x_{i}, \eta\right)\right) \int_{P_{K}^{m_{0}}} \Psi\left(\lambda \frac{d p}{d x}(x, \eta) x\right) d x=0$, hence $I_{\eta}(p, \phi)(x)=0$.

Now suppose $p \in H(X)$ (we drop the parameter in $K^{r}$ for the moment), $\phi \in S(X)$, and $\operatorname{grad} p\left(x_{0}\right)=0$ for some $x_{0} \in \operatorname{supp}(\phi)$. If $D^{2} p\left(x_{0}\right)$ is non-degenerate, we have a $p$-adic stationary phase formula, analogous to the classical case [G-S, p. 6].

Proposition 1.2. Let $X \subseteq K^{n}$ be open, $p \in H(X)$, and $\phi \in S(X)$. Suppose $\left\{x_{i}\right\}_{i \in I}$ is the set of critical points of $p$ in $\operatorname{supp}(\phi)$, and $D^{2} p\left(x_{i}\right)$ is non-degenerate for each $i \in I$. Then if $|\lambda|$ is sufficiently large,

$$
I(p, \phi)(\lambda)=|\lambda|^{-n / 2} \sum_{i \in I} c\left(p, x_{i}\right) \phi\left(x_{i}\right)
$$

for $\lambda \in\left(K^{\times}\right)^{2}$, where

$$
c\left(p, x_{i}\right)=\gamma \Psi\left(p\left(x_{i}\right)\right)\left|\operatorname{det}\left(D^{2} p\left(x_{i}\right)\right)\right|^{-1 / 2},
$$

$\gamma$ a complex eighth root of unity. 
Proof. First note that the Morse Lemma holds true for $p$-adic locally analytic functions (cf. [L, p. 174]).

Thus assuming $p$ has only one critical point $x_{0} \in U=\operatorname{supp}(\phi)$, if $D^{2} p\left(x_{0}\right)$ is non-degenerate, by the Morse Lemma (and reducing $\operatorname{supp}(\phi)$ is necessary), there is a coordinate system $y$ on $U$ such that

$$
p(x)=p\left(x_{0}\right)+\langle A y(x), y(x)\rangle, \quad x \in U,
$$

where $A=D^{2} p\left(x_{0}\right)$ is symmetric, non-degenerate. Thus

$$
\begin{aligned}
I(p, \phi)(\lambda) & =\int_{X} \phi(x) \Psi\left[\lambda\left(p\left(x_{0}\right)+\langle A y(x), y(x)\rangle\right)\right] d x \\
& =\Psi\left(\lambda p\left(x_{0}\right)\right) \int_{X} \theta(y) \Psi(\lambda\langle A y, y\rangle) d y
\end{aligned}
$$

where $\theta=\phi|\operatorname{det}(d x / d y)| \in S(X)$.

By [W, p. 161], if $\Psi_{\lambda A}(x):=\Psi(\langle A \sqrt{\lambda} x, \sqrt{\lambda} x\rangle)$, then

$$
\left(\Psi_{\lambda A}\right)^{\wedge}(y)=\gamma|\operatorname{det} A|^{-1 / 2}|\lambda|^{-n / 2} \Psi\left(-\lambda^{-1}\left\langle y,{ }^{t}\left(A^{-1}\right) y\right\rangle\right),
$$

where $\gamma$ is a complex eighth root of 1 .

Applying the Fourier multiplication formula to (1.3), we then have

$$
\begin{aligned}
I(p, \phi)(\lambda)= & \Psi\left(\lambda p\left(x_{0}\right)\right) \gamma|\operatorname{det} A|^{-1 / 2}|\lambda|^{-n / 2} \\
& \cdot \int_{X} \tilde{\hat{\theta}}(y) \Psi\left(-y^{-1}\left\langle y,{ }^{t}\left(A^{-1}\right) y\right\rangle\right) d y .
\end{aligned}
$$

To evaluate the integral, note that $y \mapsto \Psi\left(-\lambda^{-1}\left\langle y,{ }^{t}\left(A^{-1}\right) y\right\rangle\right)$ is identically 1 on $\operatorname{supp}(\tilde{\hat{\theta}})$ for $|\lambda|$ sufficiently large. Thus

$$
\begin{aligned}
I(p, \phi)(\lambda) & =\Psi\left(\lambda p\left(x_{0}\right)\right) \gamma|\operatorname{det} A|^{-1 / 2}|\lambda|^{-n / 2} \int_{X} \hat{\theta}(y) d y \\
& =\Psi\left(\lambda p\left(x_{0}\right)\right) \gamma|\operatorname{det} A|^{-1 / 2}|\lambda|^{-n / 2} \phi\left(x_{0}\right),
\end{aligned}
$$

proving the proposition.

2. P-adic wave front sets. We first define the notion of wave front set for open subsets of $K^{n}$ (c.f. [Ho, p. 119-133] for the classical case).

Definition. Let $X \subseteq K^{n}$ be open, $u \in S^{\prime}(X)$, and $\Lambda$ be an open subgroup of $K^{\times}$with $\left[K^{\times}: \Lambda\right]<\infty$. We say that $u$ is $\Lambda$-smooth at $\left(x_{0}, \xi_{0}\right) \in X \times\left(K^{n}-\{0\}\right)$ if there are open neighborhods $U$ of $x_{0}$ and $V$ of $\xi_{0}$ such that for any $\phi \in S(U)$ there is a $N_{\phi}>0$ for which $|\lambda|>N_{\phi}$ 
implies that $(\phi u) \wedge(\lambda \xi)=0$ for any $\xi \in V$. The complement of the set of smooth directions of $u$ is called the $\Lambda$-wave front set of $u$, denoted $W F_{\Lambda}(u)$.

The local nature of $W F_{\Lambda}(u)$ is shown by the $p$-adic version of [Ho, Prop. 2.5.4].

Proposition 2.1. Let $X \subseteq K^{n}$ and $U \subseteq X$ be open. Then for any $u \in S^{\prime}(X)$,

$$
W F_{\Lambda}(u \mid U)=W F_{\Lambda}(u) \cap \pi_{X}^{-1}(U),
$$

where $\pi_{X}: X \times\left(K^{n}-\{0\}\right)$ is the projection onto $X$.

The following lemma notes a useful computational simplification.

Lemma 2.2. Let $X \subseteq K^{n}$ be open and $u \in S^{\prime}(X)$. Suppose $U \subseteq X$ is open, $\phi_{U}$ is the characteristic function on $U$, and $\lambda \mapsto\left(\phi_{U} u\right)^{\wedge}(\lambda \xi)$ has bounded support on $\Lambda$, uniformly for $\xi \in V, V$ some open-compact subset of $K^{n}-\{0\}$. Then $\lambda \mapsto(\phi u) \wedge(\lambda \xi)$ has bounded support on $\Lambda$, uniformly for $\xi \in V$, for any $\phi \in S(U)$.

Proof. By hypothesis there is an $N_{U}>0$ such that when $\lambda \in \Lambda$, $|\lambda|>N_{U}$ implies $\left(\phi_{U} u\right) \hat{(}(\lambda \xi)=0$ for any $\xi \in V$. If $\phi \in S(U), \operatorname{supp}(\hat{\phi})$ is compact, so since $V$ is compact, there is an $N_{V}>0$ such that $|\lambda|>N_{V}$ implies $\lambda^{-1} \eta+\xi \in V$ for $\eta \in \operatorname{supp}(\hat{\phi}), \xi \in V$. Thus $N_{\phi}=\max \left\{N_{U}, N_{V}\right\}$ and $|\lambda|>N_{\phi}$ implies $\left(\phi_{U} u\right)^{\wedge}(\eta+\lambda \xi)=\left(\phi_{U} u\right)^{\wedge}\left(\lambda\left(\lambda^{-1} \eta+\xi\right)\right)=0$ for $\eta \in \operatorname{supp}(\hat{\phi}), \xi \in V$. Hence

$$
(\phi u)^{\wedge}(\lambda \xi)=\left(\phi \cdot \phi_{U} u\right)^{\wedge}(\lambda \xi)=\int \hat{\phi}(-\eta)\left(\phi_{U} u\right)^{\wedge}(\eta+\lambda \xi) d \eta=0
$$

proving the lemma.

EXAmPLe. Suppose the residual characteristic of $K$ is not 2, let $\varepsilon_{0} \in O_{K}^{\times}$be an element of order $q-1, \Lambda$ the image of $K\left(\sqrt{\varepsilon_{0}}\right)^{\times}$in $K^{\times}$ under the map $N_{\varepsilon_{0}}: K\left(\sqrt{\varepsilon_{0}}\right) \rightarrow K, x+\sqrt{\varepsilon_{0}} y \mapsto x^{2}-\varepsilon_{0} y^{2}$, and $H_{\Lambda}$ the characteristic function of $\Lambda$. If $\Phi_{0}$ is the characteristic function on $O_{K}$ and $u=\left(\left(1-\Phi_{0}\right) H_{\Lambda}\right) \tilde{\hat{~}}$, then $W F_{\Lambda}(u)=\{(0, \xi) \in K \times(K-\{0\}): \xi \in \Lambda\}$.

The invariance of the definition of wave front set is a consequence of the following proposition. 
Proposition 2.3. Let $p \in H\left(X \times K^{r}\right)$ and $X \subseteq K^{n}$ be open. Suppose $\operatorname{grad}_{x} p\left(x_{0}, \eta_{0}\right)=\xi_{0} \neq 0$ for some $\eta_{0} \in K^{r}$, and $\left(x_{0}, \xi_{0}\right)$ is smooth for $u \in S^{\prime}(X)$. Then there are neighborhood $U_{0}$ of $x_{0}$ and $W_{0}$ of $\eta_{0}$ such that for any $\phi \in S\left(U_{0}\right)$ there exists an $N_{\phi}>0$ for which $|\lambda|>N_{\phi}, \lambda \in \Lambda$, implies

$$
\langle u, \phi \Psi(\lambda p(\cdot, \eta))\rangle=0
$$

for any $\eta \in W_{0}$.

Proof. (After [D], Prop. 1.3.2.) Suppose $u \in S^{\prime}\left(K^{n}\right)$ is smooth on the neighborhood $U_{0} \times V$ of $\left(x_{0}, \xi_{0}\right) \in T^{*} K^{n}$, and $p \in H\left(X \times K^{r}\right)$ satisfies $\operatorname{grad}_{x} p\left(x_{0}, \eta_{0}\right)=\xi_{0}$ for some $\eta_{0} \in K^{r}$. Let $\phi \in S\left(U_{0}\right)$ and $\phi^{\prime} \in S\left(K^{n}\right)$ be 1 on $\operatorname{supp}(\phi)$. Then

$$
\begin{aligned}
\langle u, \phi \Psi(\lambda p(\cdot, \eta))\rangle= & |\lambda|^{n} \int_{K^{n}}(\phi u)^{\wedge}(\lambda \xi) \\
& \times \int_{X} \phi^{\prime}(x) \Psi(\lambda(p(x, \eta)-\langle\xi, x\rangle)) d x d \xi
\end{aligned}
$$

By hypothesis there is a $M_{\phi}>0$ such that for $\lambda \in \Lambda,|\lambda|>M_{\phi}$ implies $(\phi u)^{\wedge}(\lambda \xi)=0$ for $\xi \in V$. So suppose $\xi \notin V$. Since $\operatorname{grad}_{x} p\left(x_{0}, \eta_{0}\right)=\xi_{0}$, by shrinking $U_{0}$ if necessary, we have

$$
\left|\operatorname{grad}_{x} p(x, \eta)-\xi\right| \geq \delta>0
$$

when $x \in U_{0}$, and $\eta$ is close to $\eta_{0}$. Applying Proposition 1.1 shows that $\lambda \mapsto \int_{X} \phi^{\prime}(x) \Psi(\lambda(p(x, \eta)-\langle\xi, x\rangle)) d x$ has bounded support, and we are done.

A well defined wave front set for a distribution on an analytic manifold $X$ can thus be determined using any coordinate system on $X$. Note that Proposition 2.1 and Lemma 2.2 are valid for analytic manifolds.

Definition. For $S \subseteq K^{n}$, an $S$-cone is a set $\Gamma \subseteq K^{n} \times\left(K^{n}-\{0\}\right)$ satisfying $(x, s \xi) \in \Gamma$ whenever $(x, \xi) \in \Gamma$ and $s \in S$. $S$-cones on manifolds are defined analogously.

The proof of the next theorem is straightforward.

TheOREM 2.4. Let $u \in S^{\prime}(X), X$ be an $n$-dimensional analytic $K$ manifold, and $\Lambda$ be an open subgroup of finite index in $K^{\times}$. Then

(i) $W F_{\Lambda}(u)$ is a closed $\Lambda$-cone in $T^{*} X-\{0\}$,

(ii) If $\Lambda^{\prime}$ is a open subgroup of $\Lambda$ with $\left[\Lambda: \Lambda^{\prime}\right]<\infty$, then $W F_{\Lambda}(u)=$ $\bigcup_{\tau \in \Lambda / \Lambda^{\prime}} \tau \cdot W F_{\Lambda^{\prime}}(u)$, where $\tau \cdot W F_{\Lambda}(u):=\left\{(x, \tau \xi) \in T^{*} X: \quad(x, \xi) \in\right.$ $\left.W F_{\Lambda^{\prime}}(u)\right\}$. 
(iii) Sing $\operatorname{supp}(u)=\pi_{X}\left(W F_{\Lambda}(u)\right)$, where $\pi_{X}$ is the projection $T^{*} X \rightarrow X$.

(iv) $W F_{\Lambda}\left(u_{1}+u_{2}\right) \subseteq W F_{\Lambda}\left(u_{1}\right) \cup W F_{\Lambda}\left(u_{2}\right)$, for $u_{1}, u_{2} \in S^{\prime}(X)$.

We now develop functorial properties of $W F_{\Lambda}$. First we derive some lemmas on the geometry of $\Lambda$-cones. For $S \subseteq K^{\times}$and $T \subseteq K^{n}-\{0\}$ let $\Gamma_{S}(T):=\{s \xi: s \in S, \xi \in T\}$.

If $T$ is open then $\Gamma_{S}(T)$ is open since $s \cdot U$ is a neighborhood of $s \xi$ in $\Gamma_{S}(T)$ when $U$ is a neighborhood of $\xi \in T$. Further, if $T$ is compact and $S$ closed then $\Gamma_{S}(T)$ is closed in $K^{n}-\{0\}$.

Definition. Let $\Lambda$ be a subgroup of $K^{\times}$with $\left[K^{\times}: \Lambda\right]<\infty$ and suppose the order of $\bar{\omega} \Lambda$ in $K^{\times} / \Lambda$ is $l$. Set

$$
\Sigma_{\Lambda}^{n-1}:=\bigcup_{i=0}^{l} \bar{\omega}^{i} \cdot \Sigma^{n-1} .
$$

LEMMA 2.5. If $\Gamma \subseteq K^{n}$ is a $\Lambda$-cone and $\Gamma^{1}=\Gamma \cap \Sigma_{\Lambda}^{n-1}$, then $\Gamma=$ $\Gamma_{\Lambda}\left(\Gamma^{1}\right)$.

Proof. That $\Gamma_{\Lambda}\left(\Gamma^{1}\right) \subseteq \Gamma$ is clear. If $\xi \in \Gamma$ there is an integer $m$ such that $\xi^{\prime}=\left(\bar{\omega}^{l}\right)^{m} \xi \in \Gamma^{1}$. Since $\bar{\omega}^{l} \in \Lambda, \xi=\left(\bar{\omega}^{l}\right)^{-m} \xi^{\prime} \in \Gamma$, and $\Gamma=$ $\Gamma_{\Lambda}\left(\Gamma^{1}\right)$.

LEMMA 2.6. Let $\Gamma_{1}$ and $\Gamma_{2}$ be $\Lambda$-cones, closed in $K^{n}-\{0\}$, and suppose $\Gamma_{1} \cap \Gamma_{2}=\varnothing$. Then there exists a $\Lambda$-cone $V$, open in $K^{n}$ and closed in $K^{n}-\{0\}$, such that $\Gamma_{1} \subseteq V$ and $\Gamma_{2} \subseteq V^{\prime}=K^{n}-V$.

Proof. Let $\Gamma_{1}^{1}=\Gamma_{1} \cap \Sigma_{\Lambda}^{\mathrm{n}-1}$. Since $\Gamma_{1}^{1}$ is compact and $\Gamma_{1}^{1} \subseteq K^{-n}-\Gamma_{2}$, an open set, there is a finite set $\left\{V_{\xi_{i}}\right\}_{i \in I}$ of open-closed balls $V_{\xi_{i}}$ such that $\Gamma_{1}^{1} \subseteq V^{1}=\bigcup_{i \in I} V_{\xi_{1}}$, and $V^{1} \cap \Gamma_{2}=\varnothing$. Let $V=\Gamma_{\Lambda}\left(V^{1}\right)$. Then since $V^{1}$ is open, $V$ is an open cone, and it is also closed, since $\Lambda$ is. By Lemma 2.5, $\Gamma_{1}=\Gamma_{\Lambda}\left(\Gamma_{1}^{1}\right) \subseteq \Gamma_{\Lambda}\left(V^{1}\right)=V$, and $V \cap \Gamma_{2}=\Gamma_{\Lambda}\left(V^{1}\right) \cap \Gamma_{2}=\varnothing$. Hence $\Gamma_{2}$ $\subseteq V^{\prime}=K^{n}-V$ and $V^{\prime}$ is open.

LEMMA 2.7. Let $X$ be an open subset of $K^{n}, u \in S^{\prime}(X)$, and $x_{0} \in X$. Suppose $V$ is an open-closed $\Lambda$-cone containing

$$
\Gamma=\left\{\xi \in K^{n}-\{0\}:\left(x_{0}, \xi\right) \in W F_{\Lambda}(u)\right\},
$$

and set $V^{\prime}=\left(K^{n}-\{0\}\right)-V$. Take $p \in H\left(X \times K^{r}\right)$ with $\operatorname{grad}_{x} p\left(x_{0}, \eta\right)$ $\in V^{\prime}$, for $\eta$ in some open $W_{0} \subseteq K^{r}$. Then there is an open neighborhood $U \subseteq X$ of $x_{0}$ such that $\phi \in S(U)$ implies there is an $M_{\phi}>0$ for which, if $|\eta|>M_{\phi}, \eta \in V^{\prime}$, then $\langle u, \phi \Psi(p(\cdot, \eta))\rangle=0$. 
Proof. Assume that $X, u, x_{0}, V$, and $V^{\prime}$ are as in the hypothesis, and that $p(x, n)=\langle x, \eta\rangle$, the proof for arbitrary $p$ following from Proposition 2.3.

Let $V_{1}=V \cap \Sigma_{\Lambda}^{n-1}$. Now $V_{1}$ is open-closed so $V_{1}^{\prime}=\Sigma_{\Lambda}^{n-1}-V_{1}$ is open-closed, hence compact. Since $V_{1}^{\prime}$ is contained in $K^{n}-\Gamma$, there exists a finite set $\left\{V_{\xi_{i}}\right\}_{i \in I}$ of open-closed balls such that (i) Each $V_{\xi_{i}}$ is an open-closed neighborhood of some $\xi_{i} \in V_{1}^{\prime}$ for which there exists a neighborhood $U_{\xi_{i}}$ of $x_{0}$ such that if $\phi \in S\left(U_{\xi_{i}}\right)$, there is an $M_{\phi_{t}}>0$ for which $|\lambda|>M_{\phi_{t}}$ implies that $(\phi u)^{\wedge}(\lambda \xi)=0$, for any $\xi \in V_{\xi_{i}}$, and (ii) $V_{1}^{\prime} \subseteq \cup_{i \in I} V_{\xi_{i}}$.

Let $U=\bigcap_{i \in I} U_{\xi_{1}}, \phi \in S(U) \subseteq S\left(U_{\xi_{i}}\right), M_{\phi}=\operatorname{Max}_{i \in I}\left\{M_{\phi_{1}}\right\}$, and $i \in I$. If $\xi=\lambda \xi^{\prime}, \xi^{\prime} \in V_{\xi_{i}}$, and $|\lambda| \geq M_{\phi}$, then $(\phi u)^{\hat{\phi}}(\xi)=(\phi u)^{\wedge}\left(\lambda \xi^{\prime}\right)=0$. Hence $(\phi u)^{\wedge}$ restricted to $\Gamma_{\Lambda}\left(V_{\xi_{t}}\right)$ has bounded support. By (ii), $V^{\prime}=\Gamma_{\Lambda}\left(V_{1}^{\prime}\right)$ is contained in $\bigcup_{i \in I} \Gamma_{\Lambda}\left(V_{\xi_{i}}\right)$. Hence $(\phi u)^{\wedge}$ has bounded support in $V^{\prime}$.

A locally analytic map $f: X \rightarrow Y$ between analytic $K$ manifolds $X$ and $Y$ induces a pull back $f^{*}: C^{\infty}(Y) \rightarrow C^{\infty}(X), \phi \mapsto \phi \circ f$. If $f$ is also proper ( $f^{-1}$ (compact) is compact), then the push forward $f_{*}: S^{\prime}(X) \rightarrow S^{\prime}(Y)$ of $f$ is defined by $\left\langle f_{*} u, \phi\right\rangle=\left\langle u, f^{*} \phi\right\rangle, u \in S^{\prime}(X), \phi \in S(Y)$. We want to compute relations between $W F_{\Lambda}(u), W F_{\Lambda}\left(f^{*} u\right)$ and $W F_{\Lambda}\left(f_{*} u\right)$.

We have so far defined $f^{*}$ only on $C^{\infty}(Y)$, where $W F_{\Lambda}\left(f^{*} u\right)=\varnothing$, an uninteresting case. So we next extend the definition of $f^{*}$.

Definition. For any closed $\Lambda$-cone $\Gamma \subseteq T^{*} X-\{0\}$, let $S_{\Gamma}^{\prime}(X)$ denote the set $\left\{u \in S^{\prime}(X): W F_{\Lambda}(u) \subseteq \Gamma\right\}$.

THEOREM 2.8. Let $X$ and $Y$ be analytic $m$ - and $n$-dimensional $K$-manifolds respectively, and suppose $f: X \rightarrow Y$ is locally analytic. Let $N_{f}=\{(y, \eta)$ $\in T^{*} Y-\{0\}: y=f(x)$ for some $x \in X$ and $\left.{ }^{t} D f(x) \eta=0\right\}$. Let $\Gamma^{\prime}$ be $a$ closed $\Lambda$-cone in $T^{*} Y-\{0\}$ with $N_{f} \cap \Gamma^{\prime}=\varnothing$. If $\Gamma=$ $f^{*} \Gamma^{\prime}:=\left\{\left(x,{ }^{t} D f(x) \eta\right) \in T^{*} X\right.$ : there is a $y \in Y$ with $y=f(x)$ and $(y, \eta)$ $\left.\in \Gamma^{\prime}\right\}$, then $f^{*}: C^{\infty}(Y) \rightarrow C^{\infty}(X)$ has a unique continuous extension to a map $f^{*}: S_{\Gamma^{\prime}}^{\prime}(Y) \rightarrow S_{\Gamma}^{\prime}(X)$. Moreover

$$
W F_{\Lambda}\left(f^{*} v\right) \subseteq f^{*}\left(W F_{\Lambda}(v)\right)
$$

when $v \in S_{\Gamma^{\prime}}^{\prime}(Y)$.

Proof. By Proposition 2.1 we may assume $X \subseteq K^{m}$ and $Y \subseteq K^{n}$ are open and $\operatorname{supp}(v)$ is compact, $v \in S_{\Gamma^{\prime}}^{\prime}(Y)$. 
Now $N_{f}$ and $\Gamma^{\prime}$ are closed cones, so if $N_{f} \cap \Gamma^{\prime}=\varnothing$, by Lemma 2.6 there exists an open-closed $\Lambda$-cone $V \subseteq K^{n}-0$ such that $N_{f} \subseteq V$ and $\Gamma^{\prime} \subseteq V^{\prime}=\left(K^{n}-0\right)-V$.

If $v \in S_{\Gamma^{\prime}}^{\prime}(Y)$, by Lemma 2.7 for each $y \in Y$, there is an open-compact neighborhood $U_{y}$ such that $\chi \in S\left(U_{y}\right)$ implies $\eta \mapsto(\chi v)^{\wedge}(\eta)$ has bounded support when restricted to $V$. Thus $\int_{V}(\chi v) \wedge(\eta) I_{f}(\phi)(\eta) d \eta$ converges, where

$$
I_{f}(\phi)(\eta):=\int_{X} \phi(x) \Psi(\langle f(x),-n\rangle) d x .
$$

Assume $\operatorname{supp}(v) \subseteq U_{y_{0}}$ for some $y_{0} \in Y$.

Now let $V_{1}^{\prime}=V^{\prime} \cap \Sigma_{\Lambda}^{n-1}$. Since $N_{f} \cap V^{\prime}=\varnothing$, there is a $\delta$ such that

$$
|\langle f(x),-\eta\rangle|=\left|{ }^{t} D f(x) \eta\right| \geq \delta>0
$$

for $(x, \eta) \in U \times V_{1}^{\prime}, U=f^{-1}\left(U_{y_{0}}\right)$. By Proposition 1.1, if $\phi \in S(U)$, there is a $M_{\phi}>0$ such that $|\lambda|>M_{\phi}, \lambda \in \Lambda$, implies $I_{f}(\phi)(\lambda \eta)=0$ for any $\eta \in V_{1}^{\prime}$. So if $\xi=\lambda \eta$ is an element of $V^{\prime}=\Gamma_{\Lambda}\left(V_{1}^{\prime}\right), \lambda \in \Lambda, \eta \in V_{1}^{\prime}$, then $|\lambda|>M_{\phi}$ implies $|\lambda|>M_{\phi}$ and $I_{f}(\phi)(\xi)=I_{f}(\phi)(\lambda \eta)=0$. Hence $\eta \mapsto$ $I_{f}(\phi(\eta))$ has bounded support on $V^{\prime}$, and $\int_{V^{\prime}} \hat{v}(\eta) I_{f}(\phi)(\eta) d \eta$ converges.

Thus $\int_{K^{n}} \hat{v}(\eta) I_{f}(\phi)(\eta) d \eta$ exists and defines an extension of $f^{*}$ from $C^{\infty}(Y)$ to $S_{\Gamma}^{\prime}(Y)$.

To show that we have really extended $f^{*}$ to a map $S_{\Gamma^{\prime}}^{\prime}(Y) \rightarrow S^{\prime}(X)$ and that this extension is unique, take $v \in S(Y)\left(\subseteq S_{\Gamma^{\prime}}(Y)\right.$ for any $\Gamma$, using the standard identification). Then $\hat{v} \in S(Y)$,

$$
\int_{K^{n}} \hat{v}(\eta) I_{f}(\phi)(\eta) d \eta=\int_{X}\left[\int_{K^{n}} \Psi(\langle f(x),-\eta\rangle) \hat{v}(\eta) d \eta\right] \phi(x) d x
$$

converges for any $\phi \in S(X)$, and

$$
\begin{aligned}
\int_{X}\left[\int_{K^{n}} \Psi(\langle f(x),-\eta\rangle) \hat{v}(\eta) d \eta\right] \phi(x) d x \\
=\int_{X} v(f(x)) \phi(x) d x=\int_{X} f^{*} v(x) \phi(x) d x=\left\langle f^{*} v, \phi\right\rangle .
\end{aligned}
$$

Uniqueness comes from the fact that $C^{\infty}(Y)$ is dense in $S_{\Gamma}^{\prime}(Y)$, proved by using an approximate identity as in the classical case.

Next we show that $f^{*}$ maps $S_{\Gamma^{\prime}}^{\prime}(Y)$ into $S_{\Gamma}^{\prime}(X)$ by proving that $W F_{\Lambda}\left(f^{*} v\right) \subseteq f^{*} W F_{\Lambda}(v)$ for $v \in S_{\Gamma^{\prime}}^{\prime}(Y)$. So suppose that $\left(x_{0}, \xi_{0}\right), \xi_{0}=$ ${ }^{t} D f\left(x_{0}\right) \eta_{0}$, is not an element of $f^{*} W F_{\Lambda}(v)$, that is, $\left(f\left(x_{0}\right), \eta_{0}\right)=\left(y_{0}, \eta_{0}\right)$ $\notin W F_{\Lambda}(v)$. Let $U_{y_{0}}$ and $V_{y_{0}}$ be open-closed neighborhoods of $y_{0}$ and $\eta_{0}$, respectively, such that for any $\chi \in S\left(U_{y_{0}}\right), \lambda \mapsto(\chi v)(\lambda \eta)$ has bounded support on $\Lambda$, uniformly for $\eta \in V_{y_{0}}$. We may assume that $\operatorname{supp}(v) \subseteq U_{y_{0}}$. 
Now for any $\phi \in S(X)$,

$$
\begin{aligned}
& \left(\phi f^{*} v\right)^{\wedge}(\lambda \xi)=\int_{K^{n}} \hat{v}(\eta)\left[\int_{X} \phi(x) \Psi(\langle f(x),-\eta\rangle+\langle x, \lambda \xi\rangle) d x\right] d \eta \\
& =|\lambda|^{n} \int_{K^{n}} \hat{v}(\lambda \eta)\left\{\int_{X} \phi(x) \Psi[\lambda(\langle f(x),-\eta\rangle+\langle x, \xi\rangle)] d x\right\} d \eta \\
& =|\lambda|^{n} \int_{K^{n}} \hat{v}(\lambda \eta) I_{\phi}(\lambda, \xi, \eta) d \eta
\end{aligned}
$$

where

$$
I_{\phi}(\lambda, \xi, \eta):=\int_{X} \phi(x) \Psi(\lambda p(x, \xi, \eta)) d x
$$

with

$$
p(x, \xi, \eta):=\langle f(x),-\eta\rangle+\langle x, \xi\rangle .
$$

Let $T$ denote the subset of $K^{m+n}$,

$$
\left\{(\xi, \eta):{ }^{t} D f(x) \eta=\xi, \eta \in V, f(x) \in U\right\} .
$$

For any $\Lambda$-conic open-closed neighborhood $\Omega$ of $T$ there exists a $\delta>0$ such that

$$
\left|\operatorname{grad}_{x} p(x, \xi, \eta)\right|=\left|{ }^{t} D f(x) \eta-\xi\right| \geq \delta>0,
$$

if $x \in f^{-1}(U)$ and $(\xi, \eta)$ is not in $\Omega$. Hence by Proposition $1.1, \lambda \mapsto$ $I_{\phi}(\lambda, \xi, \eta)$ has bounded support on $\Lambda$, uniformly for $(\xi, \eta) \notin \Omega$. However if $\eta \in V_{y_{0}}, \lambda \mapsto \hat{v}(\lambda, \eta)$ has bounded support for $\lambda \in \Lambda$. Hence (2.2) does too, $\left(x_{0}, \xi_{0}\right) \notin W F_{\Lambda}\left(f^{*} v\right)$, and the theorem is proved.

Next we examine $W F_{\Lambda}\left(f_{*} u\right)$.

THEOREM 2.9. If $u \in S^{\prime}(X)$ and $f: X \rightarrow Y$ is a locally analytic proper map on $\operatorname{supp}(u)$, then $f_{*} u$ is defined and

$$
W F_{\Lambda}\left(f_{*} u\right) \subseteq f_{*}\left(W F_{\Lambda}(u)\right) \cup N_{f},
$$

where $f_{*}\left(W F_{\Lambda}(u)\right):=\left\{(y, \eta) \in T^{*} Y: y=f(x)\right.$ for some $x \in X$ and $\left.\left(x,{ }^{t} D f(x) \eta\right) \in W F_{\Lambda}(u)\right\}$.

Proof. See [G, Theorem 2].

In the classical case distribution products can be shown to exist, assuming certain criteria on wave front sets [Ho, Theorem 2.5.10]. The same is true in the $p$-adic case. 
THEOREM 2.10. Let $X$ be an $n$-dimensional analytic $K$-manifold, and $\Gamma_{1}$, $\Gamma_{2} \subseteq T^{*} X-0$ be closed $\Lambda$-cones with $\Gamma_{1} \cap-\Gamma_{2}=\varnothing$. Then there is a unique continuous extension of the product on $S(X) \times S(X)$ to a product on $S_{\Gamma_{1}}^{\prime}(X) \times S_{\Gamma_{2}}^{\prime}(X)$. If $u_{i} \in S_{\Gamma_{i}}^{\prime}(X), i=1,2$, then

$$
\operatorname{supp}\left(u_{1} \cdot u_{2}\right) \subseteq \operatorname{supp}\left(u_{1}\right) \cap \operatorname{supp}\left(u_{2}\right)
$$

and

$$
W F_{\Lambda}\left(u_{1} \cdot u_{2}\right) \subseteq\left(W F_{\Lambda}\left(u_{1}\right)+W F_{\Lambda}\left(u_{2}\right)\right) \cup W F_{\Lambda}\left(u_{1}\right) \cup W F_{\Lambda}\left(u_{2}\right)
$$

Proof. The proof is analogous to the classical case. We assume $X \subseteq K^{n}$ open. If the closed cones $\Gamma_{1}$ and $-\Gamma_{2}$ are disjoint, by Lemma 2.6 there is an open-closed $\Lambda$-cone $V$ such that $\Gamma_{1} \subseteq V$ and $-\Gamma_{2} \subseteq V^{\prime}=$ $\left(K^{n}-0\right)-V$.

Take $u_{1} \in S_{\Gamma_{1}}^{\prime}(X), u_{2} \in S_{\Gamma_{2}}^{\prime}(X)$, and $\phi \in S(X)$. Since $W F_{\Lambda}\left(u_{1}\right) \cap V^{\prime}$ $=\varnothing$, by Lemma 2.7 and assuming $\operatorname{supp}(\phi)$ sufficiently small, $\xi \mapsto$ $\left(\phi^{1 / 2} u_{1}\right) \hat{\wedge}(\xi)$ has bounded support in $V^{\prime}$. Similarly, $\xi \mapsto\left(\phi^{1 / 2} u_{2}\right) \hat{(}(-\xi)$ has bounded support in $V$. Thus the integral

$$
\int_{X}\left(\phi^{1 / 2} u_{1}\right)^{\wedge}(\xi)\left(\phi^{1 / 2} u_{2}\right)^{\wedge}(-\xi) d \xi
$$

converges, defining $\left\langle u_{1} \cdot u_{2}, \phi\right\rangle$.

Now suppose $x_{0} \notin \operatorname{supp}\left(u_{1}\right)$. Let $U \subseteq X$ be an open-closed ball with $x_{0} \in U$ and $U \cap \operatorname{supp}\left(u_{1}\right)=\varnothing$. Set $W=X-U$ and suppose $\chi_{W}$ is the characteristic function on $W$. Pick $\left\{v_{n}\right\}$ and $\left\{v_{n}^{\prime}\right\}$ to be sequences of $C^{\infty}$ functions convergent to $u_{1}$ and $u_{2}$, respectively, in $S^{\prime}(X)$. Note that $\chi_{W} v_{n} \rightarrow u_{1}$. Let $w_{n}=\left(\chi_{W} v_{n}\right) v_{n}^{\prime}$, so $w_{n} \rightarrow u_{1} \cdot u_{2}$. Then if $\phi \in S(U)$,

$$
\left\langle u_{1} \cdot u_{2}, \phi\right\rangle=\lim _{n \rightarrow \infty}\left\langle w_{n} \phi\right\rangle=\lim _{n \rightarrow \infty}\left\langle v_{n} v_{b}^{\prime}, \chi_{W} \phi\right\rangle=0 \text {, }
$$

so $x_{0} \notin \operatorname{supp}\left(u_{1} \cdot u_{2}\right)$. Similarly, $\operatorname{supp}\left(u_{1} \cdot u_{2}\right) \subset \operatorname{supp}\left(u_{2}\right)$, proving (2.3).

For the proof of (2.4) see [G, pp. 241-242], whose functorial approach we have now proved to be valid for the $p$-adic case.

As noted at the end of the proof of Theorem 2.10, Theorems 2.8-2.9 on the wave front sets of pull-backs and push-forwards give us $p$-adic versions of the basic functorial machinery in [G]. The following two results on kernel maps, which we just state, can then be proved as in [G].

THEOREM 2.11. Let $X$ and $Y$ be analytic $K$ manifolds.

(i) Suppose $\kappa \in S^{\prime}(X \times Y)$ is properly supported $\left(\pi_{*} \kappa\right.$ is defined for both projections $\pi=\pi_{X}: X \times Y \rightarrow X$ and $\left.\pi=\pi_{Y}: X \times Y \rightarrow Y\right)$, and 
$v \in S(Y)$. Then the product $L_{\kappa} v=\kappa \cdot(1 \otimes v)$ is defined, inducing a map $L_{\kappa}: \quad S(Y) \rightarrow S^{\prime}(X)$, where $\left\langle L_{\kappa} v, \phi\right\rangle:=\langle\kappa \cdot(1 \otimes v), \phi \otimes 1\rangle$, and $W F_{\Lambda}\left(L_{\kappa} v\right) \subseteq \pi_{X_{*}}\left(W F_{\Lambda}(\kappa)\right)$.

(ii) If $\Gamma \subseteq T^{*} Y-0$ is a closed $\Lambda$-cone and $-\Gamma$ is disjoint from $\{(y, \eta)$ $\in T^{*} Y:((x, y),(0, \eta)) \in W F_{\Lambda}(\kappa)$ for some $\left.x \in X\right\}$, then $L_{\kappa}$ can be uniquely extended from $S(Y)$ to a continuous map $L_{\kappa}: S_{\Gamma}^{\prime}(Y) \rightarrow S^{\prime}(X)$. If $v \in S_{\Gamma}^{\prime}(Y)$, $W F_{\Lambda}\left(L_{\kappa} v\right) \subseteq R_{\kappa}^{* \prime}\left(W F_{\Lambda}(v)\right) \cup \pi_{X_{*}}\left(W F_{\Lambda}(\kappa)\right)$, where

$$
\begin{array}{r}
R_{\kappa}^{\prime *}\left(W F_{\Lambda}(v)\right):=\left\{(x, \xi) \in T^{*} X:((x, y),(\xi,-\eta)) \in W F_{\Lambda}(\kappa)\right. \\
\text { for some } \left.(y, \eta) \in W F_{\Lambda}(v)\right\} .
\end{array}
$$

Further, if $\pi_{X_{*}}\left(W F_{\Lambda}(\kappa)\right)=\varnothing$, then by Theorem 2.4(iii), Sing $\operatorname{supp}\left(L_{\kappa} v\right)$ is contained in

$$
\{x \in X:(x, y) \in \operatorname{sing} \operatorname{supp}(\kappa) \text { for some } y \in \operatorname{sing} \operatorname{supp}(v)\} .
$$

THEOREM 2.12. Let $X, Y$, and $Z$ be analytic $K$-manifolds, $\kappa_{1} \in$ $S^{\prime}(X \times Y), \kappa_{2} \in S^{\prime}(Y \times Z)$ be properly supported, and suppose $W F_{\Lambda}\left(\kappa_{1}\right) \times$ 0 is disjoint from $-\left(0 \times W F_{\Lambda}\left(\kappa_{2}\right)\right)$. Then

(i) The composition

$$
\kappa_{1} \circ \kappa_{2}:=\pi_{X \times Z_{*}}\left(\left(\kappa_{1} \otimes 1\right) \cdot\left(1 \otimes \kappa_{2}\right)\right)
$$

exists.

(ii) $W F_{\Lambda}\left(\kappa_{1} \circ \kappa_{2}\right)$ is contained in the set

$$
W F_{\Lambda}^{\prime}\left(\kappa_{1}\right) \circ W F_{\Lambda}\left(\kappa_{2}\right) \cup\left(R_{\kappa_{1}}^{*}\left(O_{Y}\right) \times O_{Z}\right) \cup\left(O_{X} \times R_{\kappa_{2 *}}\left(O_{Y}\right)\right),
$$

where

$$
\begin{aligned}
W F_{\Lambda}^{\prime}\left(\kappa_{1}\right):=\left\{((x, y),(\xi, \eta)) \in T^{*}(X \times Y):\right. \\
\left.((x, y),(\xi,-\eta)) \in W F_{\Lambda}\left(\kappa_{1}\right)\right\},
\end{aligned}
$$

$R_{\kappa_{1}}^{*}\left(O_{Y}\right):=\left\{(x, \xi) \in T^{*} X:((x, y),(\xi, 0)) \in W F_{\Lambda}\left(\kappa_{1}\right)\right.$ for some $\left.y \in Y\right\}$,

and

$R_{\kappa_{2}}\left(O_{Y}\right):=\left\{(z, \zeta) \in T^{*} Z:((y, z),(0, \zeta)) \in W F_{\Lambda}\left(\kappa_{2}\right)\right.$ for some $\left.y \in Y\right\}$.

Here $O_{X}, O_{Y}$, and $O_{Z}$ are the zero sections in $T^{*} X, T^{*} Y$, and $T^{*} Z$ respectively.

(iii) $\operatorname{supp}\left(\kappa_{1} \circ \kappa_{2}\right) \subseteq \operatorname{supp}\left(\kappa_{1}\right) \circ \operatorname{supp}\left(\kappa_{2}\right)$.

3. $P$-adic wave front sets of group representations. In this section we turn to representations $\rho$ of $p$-adic groups, defining the notion of the wave front set of $\rho$ similar to that of $[\mathbf{H}]$ for Lie groups. Our novelty 
consists not of formulation of results, but in placement of emphasis, and verification of the $p$-adic case.

As usual, $K$ is a locally compact field with discrete valuation and $\Lambda$ is an open subgroup of $K^{\times}$with $\left[K^{\times}: \Lambda\right]<\infty$. Let $G$ be a unimodular closed subgroup of $\mathrm{GL}_{n}(K)$, closed in $K^{n^{2}}$, and $d x$ be a Haar measure on $G$. We consider only unitary representations $\left(\rho, H_{\rho}\right)$ of $G$ on the Hilbert space $H_{\rho}$. (Our results are valid for a wider range of representations, but for concreteness we take $\rho$ unitary.)

Our first definition generalizes that of $W F_{\Lambda}$ given in Chapter 2 to operator valued distributions.

Definition. We say that $\rho$ is $\Lambda$-smooth at $\left(g_{0}, \xi_{0}\right) \in T^{*} G$ if for any locally analytic function $p$ on $G \times K^{r}$, there are neighborhoods $U_{0}$ of $g_{0}$ and $W_{0} \subseteq K^{r}-\{0\}$ of $\xi_{0}$ such that $\phi \in S\left(U_{0}\right)$ implies that

$$
\langle\rho, \phi \Psi(\lambda p(\cdot, \eta))\rangle=\int_{G} \phi(x) \Psi(\lambda p(x, \eta)) \rho(x) d x=0,
$$

for any $\eta \in W_{0}$, when $|\lambda|>M_{\phi}, \lambda \in \Lambda$, for some $M_{\phi}>0$. The set of $(x, \xi) \in T^{*} G$ where $\rho$ is not smooth at $(x, \xi)$ is denoted $W F_{\Lambda}(\rho)$ and called the $\Lambda$-wave front set of the representation $\rho$.

If $L_{g}(x):=g^{-1} x$ and $R_{g}(x):=x g$ for $g, x \in G$, then $L_{g}^{*}\left(W F_{\Lambda}(\rho)\right)=$ $R_{g}^{*}\left(W F_{\Lambda}(\rho)\right)=W F_{\Lambda}(\rho)$. For if $\phi \in S(G)$, and $p \in H\left(G \times K^{r}\right)$, we have

$$
\begin{aligned}
\langle\rho, \phi \Psi(\lambda p(\cdot, \eta))\rangle & =\int_{G} \phi\left(g^{-1} x\right) \Psi\left(\lambda p\left(g^{-1} x, \eta\right)\right) \rho\left(g^{-1} x\right) d x \\
& =\rho\left(g^{-1}\right)\left\langle\rho, L_{g}^{*} \phi \Psi(\lambda p(\cdot, \eta))\right\rangle,
\end{aligned}
$$

and similarly for $R_{g}^{*}$. Thus $W F_{\Lambda}(\rho)$ can be identified with an $\operatorname{Ad} G$ invariant subset of the dual $\mathrm{g}^{*}$ of the Lie algebra $\mathrm{g}$ of $G$ :

$$
W F_{\Lambda}^{0}(\rho)=\left\{\xi \in \mathfrak{g}^{*}:(e, \xi) \in W F_{\Lambda}(\rho)\right\},
$$

where we identify $T^{*} G \simeq G \times \mathfrak{g}^{*}$. If $\xi \in \mathfrak{g}^{*}-W F_{\Lambda}(\rho)$, we say that $\rho$ is $\Lambda$-smooth at $\xi$.

We give three equivalent conditions for $\rho$-smoothness, which are the $p$-adic versions of $[\mathbf{H}$, Theorem $1.4 \mathrm{i}$, vi, vii].

LEMMA 3.1. Let $\left(\rho, H_{\rho}\right)$ be a unitary representation of the unimodular p-adic group $G$ and suppose that $V \subseteq \mathrm{g}^{*}$ is open. Then the following statements are equivalent:

(i) $V \cap W F_{\Lambda}^{0}(\rho)=\varnothing$. 
(ii) If $\phi \in S(G)$ and $p \in H\left(G \times K^{r}\right)$ satisfies $\operatorname{grad}_{x} p(\eta)(\operatorname{supp}(\phi)) \subseteq$ $V$ for all $\eta$ in some open subset $W_{0}$ of $K^{r}$, then the operator $\langle\rho, \phi \psi(\lambda p(\cdot, \eta))\rangle$ is 0 for large enough $|\lambda|$, uniformly for $\eta \in W_{0}$.

(iii) There is a neighborhood $U$ of the identity element $e$ such that whenever $\phi \in S(U), p \in H\left(G \times K^{r}\right)$, and $\operatorname{grad}_{x} p(\eta)(\operatorname{supp}(\phi)) \subseteq V$ for $\eta \in W_{0}, W_{0}$ an open subset of $K^{r}$, then $\langle\rho, \phi \Psi(\lambda p(\cdot, \eta))\rangle=0$ when $|\lambda|$ is sufficiently large, uniformly for $\eta \in W_{0}$.

Next we examine the collective behavior of the scalar distributions defined by the matrix coefficients of $\rho$. Denote by $\Upsilon\left(H_{\rho}\right)$, or just $\Upsilon_{\rho}$, the set of trace class operators on $H_{\rho}$. For any $g \in G$ and $T \in \Upsilon_{\rho}, \rho(g) T$ is of trace class, and $g \mapsto \operatorname{Tr}_{\rho}(T)(g)=\operatorname{tr}(\rho(g) T)$ defines a bounded continuous function on $G$. Let $T W F_{\Lambda}(\rho)$ denote the closed $\Lambda$-cone in $T^{*} G$ :

$$
\mathrm{Cl}\left[\bigcup_{T \in \Upsilon_{\rho}} W F_{\Lambda}\left(\operatorname{Tr}_{\rho}(T)\right)\right] \quad(\mathrm{Cl}=\text { closure }) .
$$

We show that $T W F_{\Lambda}(\rho)=W F_{\Lambda}(\rho)$. The first step is to note that $T W F_{\Lambda}(\rho)$ is invariant under $L_{g}^{*}$ and $R_{g}^{*}$. This is proved for the $p$-adic case analogously to the classical proof [H, Prop. 1.1]. We may then identify $T W F_{\Lambda}(\rho)$ with an Ad $G$ invariant subset of $g^{*}$ :

$$
T W F_{\Lambda}^{0}(\rho)=\left\{\xi \in \mathfrak{g}^{*}:(e, \xi) \in T W F_{\Lambda}(\rho)\right\} .
$$

Again it is useful to have various equivalent criteria for $\Lambda$ " $T W F_{\Lambda}^{0}(\rho)$ " smoothness.

LEMMA 3.2. Let $\left(\rho, H_{\rho}\right)$ be a unitary representation of the unimodular p-adic group $G$, and $V$ be an open subset of $\mathrm{g}^{*}$. Then the following are equivalent:

(i) $V \cap T W F_{\Lambda}^{0}(\rho)=\varnothing$.

(ii) If $T \in \Upsilon_{\rho}$ and $p \in H\left(G \times K^{r}\right)$ satisfies $\operatorname{grad}_{x} p(e, \eta) \in V$ for $\eta \in W_{0}, W_{0}$ an open subset of $K^{r}$, then there is an open neighborhood $U$ of $e$ such that for any $\phi \in S(U)$ there exists an $M_{\phi}>0$ for which $|\lambda|>M_{\phi}$, $\lambda \in \Lambda$, implies $\left\langle\operatorname{Tr}_{\rho}(T), \Phi \Psi(\lambda p(\cdot, \eta))\right\rangle=0$ for any $\eta \in W_{0}$.

(iii) If $T \in \Upsilon_{\rho}, \phi \in S(G)$, and $p \in H\left(G \times K^{r}\right)$ with $\operatorname{grad}_{x} p(\eta)$. $(\operatorname{supp}(\phi)) \subseteq V$ for $\eta \in W_{0}, W_{0}$ an open subset of $K^{r}$, then $\lambda \mapsto\left\langle\operatorname{Tr}_{\rho}(T)\right.$, $\phi \Psi(\lambda p(\cdot, \eta))\rangle$ has bounded support on $\Lambda$, uniformly for $\eta \in W_{0}$.

(iv) Same as (iii) except that we require only $\phi \in S(U)$ for some fixed neighborhood $U$ of $e$.

(v) Same as (iii) except that the support of $\lambda \mapsto\left\langle\operatorname{Tr}_{\rho}(T)\right.$, $\phi \Psi(\lambda p(\cdot, \eta))\rangle$ for fixed $\phi$ and $p$ is independent of $T$. 
Again the proof for the $p$-adic case is analogous to the classical $[\mathbf{H}$, Theorem 2.4i-v].

Lemmas 3.1 and 3.2 now imply that $W F_{\Lambda}(\rho)=T W F_{\Lambda}(\rho)$.

THEOREM 3.3. $W F_{\Lambda}^{0}(\rho)=T W F_{\Lambda}^{0}(\rho)$ for any unitary representation $\rho$ of a unimodular p-adic group $G$.

Proof. (After [H].) Let $V \subseteq \mathrm{g}^{*}$ be open. We must show that $V \cap$ $T W F_{\Lambda}^{0}(\rho)=\varnothing$ iff $V \cap W F_{\Lambda}^{0}(\rho)=\varnothing$.

So suppose $V$ and $T W F_{\Lambda}^{0}(\rho)$ are disjoint. To show that $V \cap W F_{\Lambda}^{0}(\rho)$ $=\varnothing$, suppose $\phi \in S(G), p \in H\left(G \times K^{r}\right)$, and $\operatorname{grad}_{x} p(\eta)(\operatorname{supp}(\phi)) \subseteq V$ for all $\eta \in W_{0}$, for some open $W_{0} \subseteq K^{r}$. By Lemma 3.1(ii) it suffices to show that for some $M_{\phi}>0,|\lambda|>M_{\phi}, \lambda \in \Lambda$, implies that $\langle\rho, \phi \Psi(\lambda p(\cdot, \eta))\rangle=0$ for any $\eta \in W_{0}$.

Now by Lemma 3.2(v), there is a $M_{\phi}>0$ such that $|\lambda|>M_{\phi}, \lambda \in \Lambda$, implies that for all $T \in \Upsilon_{\rho}$ and $\eta \in W_{0}$,

$$
\left\langle\operatorname{Tr}_{\rho}(T), \phi \Psi(\lambda p(\cdot, \eta))\right\rangle=\operatorname{tr}[\langle\rho \cdot \phi \Psi(\lambda p(\cdot, \eta))\rangle T]=0 .
$$

Let $\left\{e_{i}\right\}_{i \in I}$ be any orthonormal basis of $H_{\rho}$. For $i, j \in I$ define $T_{i j} \in \Upsilon_{\rho}$ by setting $T_{i j} v:=\left\langle v, e_{j}\right\rangle e_{i}$ for $v \in H_{\rho}$. Then if $|\lambda|>M_{\phi}$,

$$
\begin{aligned}
\operatorname{tr}\left[\langle\rho, \phi \Psi(\lambda p(\cdot, \eta))\rangle T_{i j}\right] & =\sum_{k \in I}\left\langle\langle\rho, \phi \Psi(\lambda p(\cdot, \eta))\rangle T_{i j} e_{k}, e_{k}\right\rangle \\
& =\left\langle\langle\rho, \phi \Psi(\lambda p(\cdot, \eta))\rangle e_{i}, e_{j}\right\rangle=0 .
\end{aligned}
$$

Hence $\langle\rho, \phi \Psi(\lambda p(\cdot, \eta))\rangle=0$ when $|\lambda|>M_{\phi}$, and $V \cap W F_{\Lambda}^{0}(\rho)=\varnothing$.

Conversely, suppose $V \cap W F_{\Lambda}^{0}(\rho)=\varnothing$. By Lemma 3.1(ii), assuming $\operatorname{grad}_{x} p(\eta)(\operatorname{supp}(\phi)) \subseteq V$ for $\phi \in S(G), \eta \in W_{0}, W_{0}$ open in $K^{r}$, there is a $M_{\phi}>0$ for which $|\lambda|>M_{\phi}, \lambda \in \Lambda$, implies $\langle\rho, \phi \Psi(\lambda p(\cdot, \eta))\rangle=0$ when $\eta \in W_{0}$. But then $\left\langle\operatorname{Tr}_{\rho}(T), \phi \Psi(\lambda p(\cdot, \eta))\right\rangle=\operatorname{tr}(0 T)=0$ for $|\lambda|>$ $M_{\phi}$, for any $T \in \Upsilon_{\rho}$. Hence by Lemma 3.2(v), $V \cap T W F_{\Lambda}^{0}(\rho)=\varnothing$, and theorem is proved.

Some representations have a natural distribution and wave front set associated with them. For example, take $G$ to be semi-simple and $\left(\rho, H_{\rho}\right)$ to be a unitary representation of $G$. We say that $\rho$ is admissible if (i) every element of $H_{\rho}$ is fixed by some open subgroup of $G$, and (ii) the subspace of $H_{\rho}$ fixed by any open subgroup of $G$ is finite dimensional [Go, p. 1.2]. It follows from (ii) that for any $\phi \in S(G),\langle\rho, \phi\rangle$ is a finite rank operator. Hence

$$
\left\langle\chi_{\rho}, \phi\right\rangle=\operatorname{tr}(\langle\rho, \phi\rangle)
$$


defines a distribution $\chi_{\rho}$ on $G$ called the character of $\rho$, which is conjugation invariant. Denote by $W F_{\Lambda}^{0}\left(\chi_{\rho}\right)$ the $\operatorname{Ad} G$ invariant $\Lambda$-conical subset of $g^{*}$ :

$$
\left\{\xi \in \mathfrak{g}^{*}:(e, \xi) \in W F_{\Lambda}\left(\chi_{\rho}\right)\right\}
$$

THEOREM 3.4. If $G$ is a unimodular semi-simple p-adic group and $\rho$ is a unitary admissible representation of $G$, then $W F_{\Lambda}^{0}\left(\chi_{\rho}\right)=W F_{\Lambda}^{0}(\rho)$.

Proof. That $W F_{\Lambda}^{0}(\rho) \subseteq W F_{\Lambda}^{0}\left(\chi_{\rho}\right)$ is proved analogously to the Lie group case [H, Theorem 1.8].

To show the converse suppose that $\rho$ is smooth at $\xi_{0}, p \in H\left(G \times K^{r}\right)$ satisfies $\operatorname{grad}_{x} p\left(e, \eta_{0}\right)=\xi_{0}$, and $U_{0} \times W_{0}$ is a neighborhood of $\left(e, \eta_{0}\right)$ such that if $\phi \in U_{0},\langle\rho, \phi \Psi(\lambda p(\cdot, \eta))\rangle=0$ for any $\eta \in W_{0}$, when $|\lambda|>M_{\phi}, \lambda \in \Lambda$, for some $M_{\phi}>0$. Then $|\lambda|>M_{\Phi}$ implies that for all $\eta \in W_{0}$,

$$
\begin{aligned}
\left\langle\chi_{\rho}, \phi \Psi(\lambda p(\cdot, \eta))\right\rangle & =\operatorname{tr}\left[\int_{G} \phi(x) \Psi(\lambda p(x, \eta)) \rho(x) d x\right] \\
& =\operatorname{tr}[\langle\rho, \phi \Psi(\lambda p(\cdot, \eta))\rangle]=\operatorname{tr}(0)=0 .
\end{aligned}
$$

Hence $\chi_{\rho}$ is smooth at $\xi_{0}$.

Not surprisingly $W F_{\Lambda}(\rho)$ obeys the same elementary properties as the scalar wave front set. Proposition 2.1, Lemma 2.2, and Theorem 2.4 (i-ii) hold true with " $X$ " replaced by " $G$ ", and " $W F_{\Lambda}(u)$ " replaced by "WF $W F_{\Lambda}(\rho)$."

The result on sums of representations is actually stronger than in the case of sums of distributions on manifolds (Theorem 2.4iv).

THEOREM 3.5. Let $\left(\rho_{1}, H_{\rho}\right)$ and $\left(\rho_{2}, H_{\rho_{2}}\right)$ be unitary representation of the unimodular p-adic group $G$, and $\left(\rho_{1} \oplus \rho_{2}, H_{\rho_{1}} \oplus H_{\rho_{2}}\right)$ their direct sum. Then

$$
W F_{\Lambda}^{0}\left(\rho_{1} \oplus \rho_{2}\right)=W F_{\Lambda}^{0}\left(\rho_{1}\right) \cup W F_{\Lambda}^{0}\left(\rho_{2}\right)
$$

Proof. It suffices to show that $W F_{\Lambda}^{0}\left(\rho_{i}\right) \subseteq W F_{\Lambda}^{0}\left(\rho_{1} \oplus \rho_{2}\right), i=1,2$, containment in the converse being implied by Theorem 2.4(iv).

Now $\Upsilon_{\rho_{1}}$ can be included in $\Upsilon_{\rho_{1} \oplus \rho_{2}}$ by identifying

$$
T \leftrightarrow\left(\begin{array}{ll}
T & 0 \\
0 & 0
\end{array}\right) \in \Upsilon_{\rho_{1} \oplus \rho_{2}}, \quad T \in \Upsilon_{\rho_{1}} .
$$


Then

$$
W F_{\Lambda}\left(\operatorname{Tr}_{\rho_{1} \oplus \rho_{2}}(T)\right)=W F_{\Lambda}\left(\operatorname{Tr}_{\rho_{1}}(T)\right),
$$

for any $T \in \Upsilon_{\rho_{1}} \subseteq \Upsilon_{\rho_{1} \oplus \rho_{2}}$. Hence $T W F_{\Lambda}\left(\rho_{1}\right) \subseteq T W F_{\Lambda}\left(\rho_{1} \oplus \rho_{2}\right)$, so

$$
W F_{\Lambda}^{0}\left(\rho_{1}\right) \subseteq W F_{\Lambda}^{0}\left(\rho_{1} \oplus \rho_{2}\right)
$$

by Theorem 3.3. Similarly $W F_{\Lambda}^{0}\left(\rho_{2}\right) \subseteq W F_{\Lambda}^{0}\left(\rho_{1} \oplus \rho_{2}\right)$, proving the theorem.

The wave front set of an outer tensor product behaves as the scalar case [G, Theorem 3].

THEOREM 3.6. Let $\left(\rho_{1}, H_{\rho_{1}}\right)$ and $\left(\rho_{2}, H_{\rho_{2}}\right)$ be unitary representations of the unimodular p-adic group $G$, and suppose $\left(\rho_{1} \otimes \rho_{2}, H_{\rho_{1} \otimes \rho_{2}}\right)$ is their outer Kronecker product. Then

$$
\begin{aligned}
W F_{\Lambda}^{0}\left(\rho_{1} \otimes \rho_{2}\right) \subseteq & W F_{\Lambda}^{0}\left(\rho_{1}\right) \times W F_{\Lambda}^{0}\left(\rho_{2}\right) \cup\left(0 \times W F_{\Lambda}^{0}\left(\rho_{2}\right)\right) \\
& \cup\left(W F_{\Lambda}^{0}\left(\rho_{1}\right) \times 0\right) .
\end{aligned}
$$

Next suppose $G=G_{1}=G_{2}$. The inner Kronecker product $\rho_{1} \times \rho_{2}$ on $G$ is given by $\rho_{1} \times \rho_{2}(g):=\rho_{1}(\mathrm{~g}) \otimes \rho_{2}(g)$. It is tempting to postulate that

$$
W F_{\Lambda}\left(\rho_{1} \times \rho_{2}\right) \subseteq\left(W F_{\Lambda}\left(\rho_{1}\right)+W F_{\Lambda}\left(\rho_{2}\right)\right) \cup W F_{\Lambda}\left(\rho_{1}\right) \cup W F_{\Lambda}\left(\rho_{2}\right) .
$$

Computing coefficients of $\left\langle\rho_{1} \otimes \rho_{2}, \phi \Psi(\lambda p(\cdot, \eta))\right\rangle$ we have formally

$$
\begin{aligned}
\left\langle\left\langle\rho_{1} \otimes \rho_{2}, \phi \Psi(\lambda p(\cdot, \eta))\right\rangle u \otimes w, u^{\prime} \otimes w^{\prime}\right\rangle \\
\quad=\left\langle\int_{G} \rho_{1}(x) u \otimes \rho_{2}(x) w \phi(x) \Psi(\lambda p(x, \eta)) d x, u^{\prime} \otimes w^{\prime}\right\rangle \\
\quad=\int_{G}\left\langle\rho_{1}(x) u, u^{\prime}\right\rangle\left\langle\rho_{2}(x) w, w^{\prime}\right\rangle \phi(x) \Psi(\lambda p(x, \eta)) d x \\
\quad=\int_{G} \operatorname{Tr}_{\rho_{1}}(S)(x) \cdot \operatorname{Tr}_{\rho_{2}}(T)(x) \phi(x) \Psi(\lambda p(x, \eta)) d x,
\end{aligned}
$$

where $S(v):=\left\langle v, u^{\prime}\right\rangle u$ and $T(v):=\left\langle v, w^{\prime}\right\rangle w$, for $u \in H_{\rho_{1}}, w \in H_{\rho_{2}}, u^{\prime} \in$ $H_{\rho_{1}}^{*}$, and $w^{\prime} \in H_{\rho_{2}}^{*}$.

If $W F_{\Lambda}\left(\operatorname{Tr}_{\rho_{1}}(S)\right)$ and $-W F_{\Lambda}\left(\operatorname{Tr}_{\rho_{2}}(T)\right)$ are disjoint, (3.1) converges, as in Theorem 2.10. However, if $\rho=\rho_{1}=\rho_{2}, S=T,-1 \in \Lambda$, and $W F_{\Lambda}\left(\operatorname{Tr}_{\rho}(S)\right)=\varnothing$, then

$$
W F_{\Lambda}\left(\operatorname{Tr}_{\rho_{1}}(S)\right) \cap W F_{\Lambda}\left(\operatorname{Tr}_{\rho_{2}}(T)\right)=W F_{\Lambda}\left(\operatorname{Tr}_{\rho}(S)\right) \neq \varnothing,
$$


and we do not know if (3.1) converges. So, in general, it is not clear how to compute $W F_{\Lambda}\left(\rho_{1} \otimes \rho_{2}\right)$.

If $\left(\rho, H_{\rho}\right)$ is a unitary representation of a group $G$, and $H$ is a subgroup of $G$, then $\left(\rho \mid H, H_{\rho}\right)$ is a unitary representation of $H$. A natural question is to ask what is the relation between $W_{\Lambda}(\rho)$ and $W F_{\Lambda}(\rho \mid H)$ when $G$ is a unimodular $p$-adic group.

THEOREM 3.7. Let $H$ be a closed subgroup of the unimodular p-adic group $G$, and $\mathfrak{h}$ denote the Lie algebra of $H$. If $\iota: H \rightarrow G$ is the inclusion map and $\rho \mid H$ is the restriction to $H$ of the unitary representation $\left(\rho, H_{\rho}\right)$ of $G$, then

$$
{ }^{t} D_{\iota}(e)\left(W F_{\Lambda}^{0}(\rho)\right) \subseteq W F_{\Lambda}^{0}(\rho \mid H) .
$$

Further, if $N_{\iota}$ is disjoint from $W F_{\Lambda}^{0}(\rho)$, then

$$
{ }^{t} D_{\iota}(e)\left(W F_{\Lambda}^{0}(\rho)\right)=W F_{\Lambda}^{0}(\rho \mid H) .
$$

Proof. The proof for the Lie group case [H, Props. 1.5-6] also handles the $p$-adic case.

We conclude with non-trivial examples of representation wave front sets.

EXAMPLE 1. Let $G=K^{n}$ and $\left(\rho, L^{2}(G)\right)$ be the regular representation of $G . \rho$ is equivalent by the Fourier transform to the representation

$$
\rho(x) f(y)=\overline{\Psi(\langle x, y\rangle)} f(y), \quad x \in K^{n}, f \in L^{2}(G) .
$$

Take $\phi$ to be the characteristic function of $\left(O_{K}\right)^{n}$, and set $p(x, \eta)=\langle x, \eta\rangle$. Then

$$
\begin{aligned}
\langle\rho, \phi \Psi(\lambda & \langle\cdot, \xi\rangle)\rangle f(y)=\int_{K^{n}} \phi(x) \Psi(\lambda\langle x, \xi\rangle)(\rho(x) f)(y) d x \\
& =\int_{K^{n}} \phi(x) \Psi(\lambda\langle x, \xi\rangle) \overline{\Psi(\langle x, y\rangle)} f(y) d x=\hat{\phi}(\lambda \xi-y) f(y),
\end{aligned}
$$

and $W F_{\Lambda}^{0}(\rho)=K^{n}-0$.

EXAMPle 2. Suppose $G$ is nilpotent. The computation is the same as $[\mathbf{H}$, p. 131], since the Lie and $p$-adic theory are the same [K]. To each irreducible unitary representation $\left(\rho, H_{\rho}\right)$ there is associated an orbit $\Omega_{\rho}=\left\{\operatorname{Ad}^{*}(x) \nu_{0}: x \in G\right\}$ for some $\nu_{0} \in \mathfrak{g}^{*}$, such that

$$
\begin{aligned}
\left\langle\chi_{\rho}, \phi\right\rangle & =\operatorname{tr}\left(\int_{G} \phi(x) \rho(x) d x\right) \\
& =\int_{\Omega_{\rho}} \hat{\phi}(\nu) d \Omega(\nu), \quad \phi \in S(G),
\end{aligned}
$$


where

$$
\hat{\phi}(\nu)=\int_{\mathfrak{g}^{*}} \phi(\exp (X)) \Psi(\langle X, \nu\rangle) d X,
$$

and $d \Omega$ is an invariant measure on $\Omega_{\rho}$. Note that exp is defined on all of since $\exp (X)$ is a finite sum and $X \in \mathrm{g}^{*}$.

We use (3.2) to compute $W F_{\Lambda}^{0}(\rho)$. Let $\phi \in S(G)$ and $p \in H\left(G \times K^{r}\right)$. Then

$$
\begin{aligned}
& \left\langle\chi_{\rho}, \phi \Psi(\lambda p(\cdot, \xi))\right\rangle=\int_{\Omega_{\rho}}[\phi \Psi(\lambda p(\cdot, \xi))]^{\wedge}(\nu) d \Omega(\nu) \\
& \quad=\int_{\Omega_{\rho}}\left\{\int_{\mathfrak{g}^{*}} \phi(\exp (X)) \Psi[\lambda p(\exp (X), \eta)] \Psi(\langle X, \nu\rangle) d K\right\} d \Omega(\nu) \\
& \quad=\int_{\Omega_{\rho}}\left\{\int_{\mathfrak{g}^{*}} \phi(\exp (X)) \Psi[\lambda p(\exp (X), \eta)+\langle X, \nu\rangle] d X\right\} d \Omega(\nu)
\end{aligned}
$$

If we take $p(\exp (X), \xi)=\langle X, \xi\rangle,(3.3)$ becomes

$$
\int_{\Omega_{\rho}} \hat{\phi}(\nu+\lambda \xi) d \Omega(\nu)
$$

Hence $\left\langle\chi_{\rho}, \phi \Psi(\lambda p(\cdot, \xi))\right\rangle$ has bounded support on $\Lambda$ iff $\Omega_{\rho}+\lambda \xi$ is disjoint from $\operatorname{supp}(\hat{\phi})$ for $|\lambda|$ sufficiently large, $\lambda \in \Lambda$. Thus by Theorem 3.3, $\xi \notin W F_{\Lambda}^{0}(\rho)$ iff there is a neighborhood $U \subseteq g^{*}$ of 0 such that $\Omega_{\rho}+\lambda \xi \cap U=\varnothing$ for $|\lambda|$ sufficiently large.

EXAMPLE 3. Take $G$ to be a unimodular semi-simple $p$-adic group. Let $\left(\rho, H_{\rho}\right)$ be an irreducible admissible representation of $G$. Then $\chi_{\rho}$ is a locally summable function of $G$, and is locally constant on an open, dense subset $G^{\prime}$ of $G$. $\chi_{\rho}$ is not $C^{\infty}$ at $g=e\left(\notin G^{\prime}\right)$. However, there is a neighborhood $V$ of 0 in $g$, and constants $c_{\Omega}$ such that

$$
\chi_{\rho}(\exp (X))=\sum_{\Omega} c_{\Omega} \cdot \hat{\mu}_{\Omega}(X), \quad X \in V,
$$

the (finite) sum being over the nilpotent orbits in $\Omega$ in $\mathrm{g}^{*}$ [HC, p. 180]. Here the locally summable functions $\hat{\mu}_{\Omega}$ are defined as follows [R]: Fix $X_{0} \in \mathrm{g}$ and let $\Omega_{X_{0}}=\left\{\operatorname{Ad}(x) X_{0}: x \in G\right\}$. If $G_{X_{0}}$ is the stabilizer of $X_{0}$, then $G_{X_{0}}$ is unimodular and $G / G_{X_{0}}$ has an invariant measure $d \Omega$, and we define

$$
\left\langle\mu_{\Omega}, \phi\right\rangle=\int_{G / G_{X_{0}}} \phi\left(\operatorname{Ad}(x) X_{0}\right) d \Omega(x), \quad \phi \in S(\mathfrak{g})
$$


Note that we have identified $g$ with $g^{*}$ using a fixed non-degenerate, symmetric, $G$-invariant, bi-linear form $\langle$,$\rangle .$

Using (3.4) for $\chi_{\rho}$ on $W=\exp (V)$, if $\phi \in S(W)$, we have that for any $p \in H\left(G \times K^{r}\right)$,

$$
\begin{aligned}
\left\langle\chi_{\rho}, \phi \Psi(\lambda p(\cdot, \xi))\right\rangle & =\int_{G} \phi(x) \Psi(\lambda p(x, \xi)) \chi_{\rho}(x) d x \\
& =\int_{V} \phi^{\prime}(X) \Psi[\lambda p(\exp (X), \xi)] \chi_{\rho}(\exp (X)) d x \\
& =\int_{V} \phi^{\prime}(X) \Psi[\lambda p(\exp (X), \xi)]\left\{\sum_{\Omega} c_{\Omega} \cdot \hat{\mu}_{\Omega}(X)\right\} d X \\
& =\sum_{\Omega \text { nilpotent }} c_{\Omega} \cdot\left\langle\hat{\mu}_{\Omega}, \phi^{\prime} \Psi(\lambda p(\cdot, \xi))\right\rangle,
\end{aligned}
$$

where $\phi^{\prime} \in S(V)$ and $p^{\prime}(\cdot, \xi)=\exp ^{*} p(\cdot, \xi)$. Thus by Prop. 2.4(iv), $W F_{\Lambda}^{0}\left(\chi_{\rho}\right)$ is contained in

$$
\bigcup_{\Omega \text { nilpotent }} W F_{\Lambda}^{0}\left(\hat{\mu}_{\Omega}\right)
$$

where

$$
W F_{\Lambda}^{0}\left(\hat{\mu}_{\Omega}\right)=\left\{\xi \in g:(e, \xi) \in W F_{\Lambda}\left(\hat{\mu}_{\Omega}\right)\right\} .
$$

So let $\Omega \subseteq \mathfrak{g}$ be a nilpotent orbit generated by $X_{0} \in \mathfrak{g}$. For $\phi^{\prime} \in S(V)$, by (3.5),

$$
\begin{aligned}
& \left\langle\hat{\mu}_{\Omega}, \phi^{\prime} \Psi\left(\lambda p^{\prime}(\cdot, \xi)\right)\right\rangle=\left\langle\mu_{\Omega},\left[\phi^{\prime} \Psi\left(\lambda p^{\prime}(\cdot, \xi)\right)\right]^{\wedge}\right\rangle \\
& \quad=\int_{G / G_{X_{0}}}\left[\phi^{\prime} \Psi\left(\lambda p^{\prime}(\cdot, \xi)\right)\right]^{\wedge}\left(\operatorname{Ad}(x) X_{0}\right) d \Omega(x) \\
& \quad=\int_{G / G_{X_{0}}}\left[\int_{\mathfrak{g}} \phi^{\prime}(Y) \Psi\left(\lambda p^{\prime}(Y, \xi)\right) \Psi\left(\left\langle\operatorname{Ad}(x) X_{0}, Y\right\rangle\right) d Y\right] d \Omega(x)
\end{aligned}
$$

Letting $p^{\prime}(Y, \xi)=\langle Y, \xi\rangle,(3.6)$ becomes

$$
\begin{aligned}
\int_{G / G_{X_{0}}} & {\left[\int \phi^{\prime}(Y) \Psi\left(\left\langle\lambda \xi+\operatorname{Ad}(x) X_{0}, Y\right\rangle\right) d Y\right] d \Omega(x) } \\
= & \int_{G / G_{X_{0}}} \hat{\phi}^{\prime}\left(\lambda \xi+\operatorname{Ad}(x) X_{0}\right) d \Omega(x) .
\end{aligned}
$$

But this is 0 for large $|\lambda|$ iff $\lambda \xi+\Omega$ is disjoint from $\operatorname{supp}(\hat{\phi})$ for $|\lambda|$ sufficiently large. Thus by Theorem $3.3, \xi \notin W F_{\Lambda}^{0}(\rho)$ iff there is an open 
neighborhood $U \subseteq \mathrm{g}$ of 0 such that $N+\lambda \xi \cap U=\varnothing$ for $|\lambda|$ sufficiently large, where $N$ is the set of nilpotent elements of $g$.

Acknowledgment. The author thanks Professor H. Jacquet for his encouragement and useful discussions.

\section{REFERENCES}

[D] J. Duistermaat, Fourier Integral Operators, Courant Institute of Mathematical Science, New York (1973).

[G] A. Gabor, Remarks on the wave front set of a distribution, Trans. Amer. Math. Soc., 170 (1972), 239-244.

[Go] R. Godement, Notes on Jacquet-Langlands Theory, Inst. of Advanced Study Notes, Princeton, New Jersey (1970).

[G-S] V. Guillemin and S. Sternberg, Geometric Asymptotics, Math. Surv., 14 Amer. Math. Soc., Providence, R.I. (1977).

[HC] Harish-Chandra, The Characters of Reductive P-Adic Groups, Contributions to Algebra, Academic Press, New York (1977).

[Ho] L. Hormander, Fourier integral operators I, Acta. Math., 27 (1971), 79-183.

[H] R. Howe, Wave Front Sets of Representations of Lie Groups, Automorphic Forms, Representation Theory, and Arithmetic, Tata Inst., Bombay (1979).

[K] A. A. Kirillov, Unitary Representations of Nilpotent Lie Groups, Russian Math. Sur., 17 No. 4, (1962), 53-103.

[L] S. Lang, Analysis II, Addison-Wesley, Reading, MA, (1969).

[R] R. R. Rao, Orbital integrals in reductive groups, Amer. J. Math., 96 (1972), 505-510.

[W] A. Weil, Sur Certains Groups d'Operateurs Unitaires, Acta Math., 111 (1964).

Received June 15, 1982. A portion of this work was supported by the U.S. Department of Energy Contract No. DE-AC02-76-CHO-3073.

Plasma Physics Laboratory

PRINCETON UNIVERSITY

PRINCETON, NJ 08544 



\title{
PACIFIC JOURNAL OF MATHEMATICS EDITORS
}

\author{
DONALD BABBITT (Managing Editor) \\ University of California \\ Los Angeles, CA 90024 \\ J. DugundJI \\ University of Southern California \\ Los Angeles, CA 90089-1113 \\ R. FINN \\ Stanford University \\ Stanford, CA 94305 \\ Hermann FlaschKa \\ University of Arizona \\ Tucson, AZ 85721
}

\author{
C. C. MOORE \\ University of California \\ Berkeley, CA 94720 \\ ARTHUR OGUS \\ University of California \\ Berkeley, CA 94720 \\ Hugo Rossi \\ University of Utah \\ Salt Lake City, UT 84112 \\ H. SAMELSON \\ Stanford University \\ Stanford, CA 94305
}

\begin{tabular}{|c|c|c|c|c|}
\hline \multicolumn{5}{|c|}{ ASSOCIATE EDITORS } \\
\hline R. ARENS & $\begin{array}{l}\text { E. F. BECKENBACH } \\
(1906-1982)\end{array}$ & NeumanN & F. Wolf & K. YosHIDA \\
\hline \multicolumn{5}{|c|}{ SUPPORTING INSTITUTIONS } \\
\hline \multirow{2}{*}{\multicolumn{2}{|c|}{$\begin{array}{l}\text { UNIVERSITY OF ARIZONA } \\
\text { UNIVERSITY OF BRITISH COLUMBIA }\end{array}$}} & \multicolumn{3}{|c|}{ UNIVERSITY OF OREGON } \\
\hline & & \multirow{2}{*}{\multicolumn{3}{|c|}{ UNIVERSITY OF SOUTHERN CALIFORNIA }} \\
\hline \multirow{2}{*}{\multicolumn{2}{|c|}{$\begin{array}{l}\text { CALIFORNIA INSTITUTE OF TECHNOLOGY } \\
\text { UNIVERSITY OF CALIFORNIA }\end{array}$}} & & & \\
\hline & & \multicolumn{3}{|c|}{$\begin{array}{l}\text { STANFORD UNIVERSITY } \\
\text { UNIVERSITY OF HAWAII }\end{array}$} \\
\hline & \multicolumn{3}{|c|}{ UNIVER SITY OF TOKYO } \\
\hline \multicolumn{2}{|c|}{$\begin{array}{l}\text { MONTANA STATE UNIVERSITY } \\
\text { UNIVERSITY OF NEVADA, RENO }\end{array}$} & \multicolumn{3}{|l|}{ UNIVER } \\
\hline \multicolumn{2}{|c|}{ NEW MEXICO STATE UNIVERSITY } & \\
\hline \multicolumn{2}{|c|}{ OREGON STATE UNIVERSITY } & \multicolumn{3}{|c|}{$\begin{array}{l}\text { WASHINGTON STATE UNIVERSITY } \\
\text { UNIVERSITY OF WASHINGTON }\end{array}$} \\
\hline
\end{tabular}

The Supporting Institutions listed above contribute to the cost of publication of this Journal, but they are not owners or publishers and have no responsibility for its content or policies.

\begin{abstract}
Mathematical papers intended for publication in the Pacific Journal of Mathematics should be in typed form or offset-reproduced (not dittoed), double spaced with large margins. Please do not use built up fractions in the text of the manuscript. However, you may use them in the displayed equations. Underline Greek letters in red, German in green, and script in blue. The first paragraph must be capable of being used separately as a synopsis of the entire paper. In particular it should contain no bibliographic references. Please propose a heading for the odd numbered pages of less than 35 characters. Manuscripts, in triplicate, may be sent to any one of the editors. Please classify according to the scheme of Math. Reviews, Index to Vol. 39. Supply name and address of author to whom proofs should be sent. All other communications should be addressed to the managing editor, or Elaine Barth, University of California, Los Angeles, California 90024.

There are page-charges associated with articles appearing in the Pacific Journal of Mathematics. These charges are expected to be paid by the author's University, Government Agency or Company. If the author or authors do not have access to such Institutional support these charges are waived. Single authors will receive 50 free reprints; joint authors will receive a total of 100 free reprints. Additional copies may be obtained at cost in multiples of 50 .
\end{abstract}

The Pacific Journal of Mathematics is issued monthly as of January 1966. Regular subscription rate: $\$ 190.00$ a year (5 Vols., 10 issues). Special rate: $\$ 66.00$ a year to individual members of supporting institutions.

Subscriptions, orders for numbers issued in the last three calendar years, and changes of address should be sent to Pacific Journal of Mathematics, P.O. Box 969, Carmel Valley, CA 93924, U.S.A. Old back numbers obtainable from Kraus Periodicals Co., Route 100, Millwood, NY 10546.

The Pacific Journal of Mathematics at P.O. Box 969, Carmel Valley, CA 93924 (ISSN 0030-8730) publishes 5 volumes per year. Application to mail at Second-class postage rates is pending at Carmel Valley, California, and additional mailing offices. Postmaster: Send address changes to Pacific Journal of Mathematics, P.O. Box 969, Carmel Valley, CA 93924.

\section{PUBLISHED BY PACIFIC JOURNAL OF MATHEMATICS, A NON-PROFIT CORPORATION}




\section{Pacific Journal of Mathematics}

\section{Vol. 116, No. 2 December, 1985}

Richard Arens, Reducing the order of the Lagrangean for a classical field in curved space-time ................................ 209

Shiferaw Berhanu, W. Wistar (William) Comfort and James Dolan Reid, Counting subgroups and topological group topologies $\ldots \ldots \ldots \ldots \ldots 217$

Michael James Cambern, Isomorphisms of spaces of norm-continuous



Gerald Arthur Edgar and Jun Feng Zhao, The ordering structure on

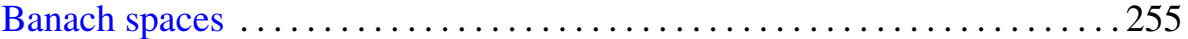

Irving Leonard Glicksberg, An analogue of Liapounoff's convexity theorem for Birnbaum-Orlicz spaces and the extreme points of their unit

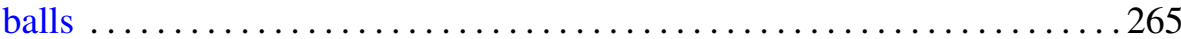

Daniel Heifetz, $p$-adic oscillatory integrals and wave front sets . ........285

José E. Pantoja, Liftings of supercuspidal representations of $\mathrm{Gl}_{2} \ldots \ldots \ldots 307$

Steven C. Pinault, An isoperimetric inequality for surfaces stationary with respect to an elliptic integrand and with at most three boundary

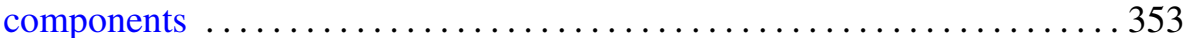

Georges Pinczon, Nonlinear multipliers and applications .............. 359 